\title{
Time crystals from minimum time uncertainty
}

\author{
Mir Faizal ${ }^{1, a}$, Mohammed M. Khalil ${ }^{2, b}{ }^{2, b}$, Saurya Das ${ }^{3, c}$ \\ ${ }^{1}$ Department of Physics and Astronomy, University of Waterloo, Waterloo, ON N2L 3G1, Canada \\ ${ }^{2}$ Department of Electrical Engineering, Alexandria University, Alexandria 12544, Egypt \\ ${ }^{3}$ Department of Physics and Astronomy, University of Lethbridge, 4401 University Drive, Lethbridge, AB T1K 3M4, Canada
}

Received: 2 October 2015 / Accepted: 5 January 2016 / Published online: 21 January 2016

(C) The Author(s) 2016. This article is published with open access at Springerlink.com

\begin{abstract}
Motivated by the Generalized Uncertainty Principle, covariance, and a minimum measurable time, we propose a deformation of the Heisenberg algebra and show that this leads to corrections to all quantum mechanical systems. We also demonstrate that such a deformation implies a discrete spectrum for time. In other words, time behaves like a crystal. As an application of our formalism, we analyze the effect of such a deformation on the rate of spontaneous emission in a hydrogen atom.
\end{abstract}

\section{Introduction}

The Heisenberg uncertainty principle predicts that the position of a particle can, in principle, be measured as accurately as one wants if its momentum is allowed to remain completely uncertain. However, most approaches to quantum gravity predict the existence of a minimum measurable length scale, usually the Planck length. There are also strong indications from black hole physics and other sources for the existence of a minimum measurable length [1-3]. This is because the energy needed to probe spacetime below the Planck length scale exceeds the energy needed to produce a black hole in that region of spacetime. Similarly, string theory also predicts a minimum length, as strings are the smallest probes [4-8]. Also in loop quantum gravity there exists a minimum measurable length scale, which turns the big bang into a big bounce [9].

The existence of a minimum measurable length scale in turn requires the modification of the Heisenberg uncertainty principle into a Generalized Uncertainty Principle (GUP) [47]; there is a corresponding deformation of the Heisenberg algebra to include momentum-dependent terms and a modified coordinate representation of the momentum operators

\footnotetext{
a e-mail: f2mir@uwaterloo.ca

b e-mail: moh.m.khali1@gmail.com

c e-mail: saurya.das@uleth.ca
}

$[8,10-15]$. It may be noted that a different kind of deformation of the Heisenberg algebra occurs due to Doubly Special Relativity (DSR) theories, which postulate the existence of a universal energy scale (the Planck scale) [16-18]. These are also related to the idea of discrete spacetime [19], spontaneous symmetry breaking of Lorentz invariance in string field theory [20], spacetime foam models [21], spin-network in loop quantum gravity [22], non-commutative geometry [23-25], ghost condensation in perturbative quantum gravity [26], and Horava-Lifshitz gravity [27]. It may be noted that DSR has been generalized to curved spacetime and the resultant theory is called gravity's rainbow [28-33]. It is interesting to note that the deformation from DSR and the deformation from GUP can be combined into a single consistent deformation of the Heisenberg algebra [34].

A number of interesting quantum systems have been studied using this deformed algebra, such as the transition rate of ultra-cold neutrons in gravitational field [35], the Lamb shift and Landau levels [36]. There has been another interesting result derived from this deformed algebra, which shows that space needs to be a discrete lattice, and only multiples of a fundamental length scale (normally taken as the Planck length) can be measured [37]. Note that minimum length does not automatically imply discrete lengths, or vice versa. Motivated by this result, in this paper we analyze the deformation of the algebra and the subsequent Schrödinger equation consistent with the existence of a minimum time, and demonstrate that it leads to a discretization of time as well. It may be noted that discretization of time had also been predicted from a deformed version of the Wheeler-DeWitt equation [38]. The discretization of time, and the related breakdown of time reparametrization invariance of a system resembles a crystal lattice in time. Time crystals have been studied recently using a very different physical motivation, e.g. analyzing superconducting rings, and the spontaneous breakdown of time-translation symmetry in classical and quantum systems [39-43]. 


\section{Observable time}

In this section, we review the work done on viewing time as a quantum mechanical observable. It is well known that time cannot be represented as a self-adjoint operator [44]. This is because the Hamiltonian with a semi-bounded spectrum does not admit a group of shifts which can be generated from canonically conjugate self-adjoint operators. However, von Neumann had suggested that restricting quantum mechanics to self-adjoint operators could be quite limiting [45]. In fact, it was demonstrated by von Neumann that the momentum operator for a free particle bounded by a rigid wall at $x=0$ is not a self-adjoint operator but only a maximal Hermitian operator. This situation is similar to the time being defined as an observable.

It has been demonstrated that under certain conditions time can be viewed as a quantum mechanical observable [46-50]. This is because it is possible to use symmetric nonself-adjoint operators that satisfy the commutation relation [51,52],

$$
[t, H]=-i \hbar
$$

In this formalism, observables are viewed as positive operator valued measures. Now for a system with Hamiltonian $H$ the map $b \rightarrow e^{i H b}$ constitutes a unitary representation of the time translation group. Thus, the positive operator valued $B$, with $\theta \rightarrow B(\theta)$, represents a time observation of the system, and it will satisfy $e^{i H b} B(\theta) e^{-i H b}=B(\theta-b)$. So for a time observable $B$, it is possible to define a symmetric time operator $t=\int t d B(t)$. This operator will not be self-adjoint. However, self-adjointness is not essential for calculating probabilities associated with the system. So, for any experiment the probability measure $\theta \rightarrow p(\theta)$ can be associated with the states $\rho$ by defining $p(\theta)=\operatorname{tr}[\rho B(\theta)]$, where $\theta \rightarrow B(\theta)$ is a positive operator valued measure [46]. Thus, it is possible to formally define time as an observable by using a maximal Hermitian (but non-self-adjoint) operator for time.

It is this definition of time that we will use when formally deforming the commutation relation. What we intend to do in this paper is to deform this formal definition of time to be consistent with the existence of a minimum measurable time interval. Mathematically this situation will be similar to the GUP deformation of the usual Heisenberg algebra. Physically observable time can be defined by defining an observable with reference to the evolution of some non-stationary quantity, if events are characterized by of a specific values of this quantity [46]. Such a non-stationary quantity could be the tunneling time for particles. Then the existence of a minimum measurable time interval will constitute a lower bound on such measurements. The existence of a lower bound on such measurements will effect the measurement of tunneling time for particles. In fact, such system have been analyzed by considering time as an observable [47-50]. Even though such an analysis is important, we will concentrate on another problem in this paper. We will analyze the deformation of commutator between the Hamiltonian and time, and demonstrate that such a deformation can lead to the existence of a discrete spectrum for time.

\section{Minimum time}

We start with the modified Heisenberg algebra, the modified expression of the momentum operator in position space, and the GUP consistent with all theoretical models, correct to $\mathscr{O}\left(\alpha^{2}\right)$. In this paper, we use units in which $c=1$. We have

$$
\begin{aligned}
{\left[x^{i}, p_{j}\right]=} & i \hbar\left[\delta_{j}^{i}-\alpha\left|p^{k} p_{k}\right|^{1 / 2} \delta_{j}^{i}+\alpha\left|p^{k} p_{k}\right|^{-1 / 2} p^{i} p_{j}\right. \\
& \left.+\alpha^{2} p^{k} p_{k} \delta_{j}^{i}+3 \alpha^{2} p^{i} p_{j}\right] \\
p_{i}= & -i \hbar\left(1-\hbar \alpha \sqrt{-\partial^{j} \partial_{j}}-2 \hbar^{2} \alpha^{2} \partial^{j} \partial_{j}\right) \partial_{i}
\end{aligned}
$$

where $\alpha=\alpha_{0} \ell_{P l} / \hbar$, and $\ell_{P l}$ is the Planck length. It has been suggested that the parameter $\alpha_{0}$ could be situated at an intermediate scale between the electroweak scale and the Planck scale, and this could have measurable consequences in the near future [36]. However, if such a deformation parameter exists, then it would be universal for all processes. This is because it would be the parameter controlling low energy phenomena occurring because of quantum gravitational effects, and as gravity affects all systems universally, we expect this parameter also to universally deform all quantum mechanical systems. Also the apparent non-local nature of operators in Eq. (3) above poses no problem in one dimension (space or time). In more than one dimensions, the issue was tackled by using the Dirac equation [34]. It is also possible to deal with these non-local derivatives, in more than one dimensions, using the theory of harmonic extension of functions [56,57]. The modified Heisenberg algebra is consistent with the following GUP, in one dimension [36]:

$$
\begin{aligned}
& \Delta x \Delta p \geq \frac{\hbar}{2}\left[1-2 \alpha\langle p\rangle+4 \alpha^{2}\left\langle p^{2}\right\rangle\right] \\
& \geq \frac{\hbar}{2}\left[1+\left(\frac{\alpha}{\sqrt{\left\langle p^{2}\right\rangle}}+4 \alpha^{2}\right) \Delta p^{2}\right. \\
&\left.+4 \alpha^{2}\langle p\rangle^{2}-2 \alpha \sqrt{\left\langle p^{2}\right\rangle}\right] .
\end{aligned}
$$

One way to arrive at the temporal deformation of the commutator is to use the principle of covariance and propose the following deformation spacetime commutators: 


$$
\begin{aligned}
& {\left[x^{\mu}, p_{v}\right]=} i \hbar\left[\delta_{v}^{\mu}-\alpha\left|p^{\rho} p_{\rho}\right|^{1 / 2} \delta_{v}^{v}+\alpha\left|p^{\rho} p_{\rho}\right|^{-1 / 2} p^{\mu} p_{v}\right. \\
&\left.+\alpha^{2} p^{\rho} p_{\rho} \delta_{v}^{\mu}+3 \alpha^{2} p^{\mu} p_{v}\right] \\
& p_{\mu}=-i \hbar\left(1-\hbar \alpha \sqrt{-\partial^{v} \partial_{v}}-2 \hbar^{2} \alpha^{2} \partial^{v} \partial_{v}\right) \partial_{\mu} .
\end{aligned}
$$

Even though we could study a temporally deformed system by using the temporal part of this covariant algebra, we will only deform the commutation relation between energy and time. This is because the deformation of the spatial part of the Heisenberg algebra has been thoroughly analyzed [3437], and here we would like to analyze the effect of temporal deformation alone on a system. We will also simplify our analysis by only deforming the relation between time and Hamiltonian of a system. This deformation will be different from the temporal part of the deformed covariant algebra. It may be noted that such a deformation only makes sense if we view time as a quantum mechanical observable. Therefore we first define the original commutator of this observable time with Hamiltonian as $[t, H]=-i \hbar[51,52]$. Then we deform this commutator of the observable time with Hamiltonian to

$[t, H]=-i \hbar[1+f(H)]$,

where $f(H)$ is a suitable function of the Hamiltonian of the system. Thus, the temporal part of Eq. (6) yields the modified Schrödinger equation

$H \psi=i \hbar \partial_{t} \psi+\hbar^{2} \alpha \partial_{t}^{2} \psi$

As can be seen from the above, this deformation of quantum Hamiltonian will produce corrections to all quantum mechanical systems. The temporal part also implies the following time-energy uncertainty:

$$
\begin{aligned}
\Delta t \Delta E \geq & \frac{\hbar}{2}\left[1-2 \alpha\langle E\rangle+4 \alpha^{2}\left\langle E^{2}\right\rangle\right] \\
\geq & \frac{\hbar}{2}\left[1+\left(\frac{\alpha}{\sqrt{\left\langle E^{2}\right\rangle}}+4 \alpha^{2}\right) \Delta E^{2}\right. \\
& \left.+4 \alpha^{2}\langle E\rangle^{2}-2 \alpha \sqrt{\left\langle E^{2}\right\rangle}\right] .
\end{aligned}
$$

\section{Time crystals}

The spatially deformed Heisenberg algebra has been used for analyzing a free particle in a box [37]. The boundary conditions which were used for analyzing this system were $\psi(0)=0$ and $\psi(L)=0$, where $L$ was the length of the box. It was demonstrated that the length of the box was quantized because of the spatial deformation of the Heisenberg algebra. As this particle was used as a test particle to measure the length of the box, this implied that space itself was quantized.
The same argument can be now used for the temporal deformation. This can be done by taking the temporal analog of the particle in a box. The boundary conditions for this system can be written as $\psi(0)=0$ and $\psi(T)=0$, where $T$ is a fixed interval of time. This is the temporal analog of a particle in a box, and the particle in this case is a test particle which measures the interval of time. Now we will demonstrate that in this case the interval of time has to be quantized. As this particle is a test particle used to measure this interval of time, we can argue that time itself is quantized.

The temporal part of the deformed Schrödinger equation to first order in $\alpha$ is given by

$i \hbar \partial_{t} \psi+\hbar^{2} \alpha \partial_{t}^{2} \psi=E \psi$

and it has the solution

$\psi(t)=A e^{\frac{-i t(1+\sqrt{1-4 E \alpha})}{2 \alpha \hbar}}+B e^{\frac{-i t(1-\sqrt{1-4 E \alpha})}{2 \alpha \hbar}}$.

Applying the boundary condition $\psi(0)=0$ leads to $B=$ $-A$, and the second boundary condition $\psi(T)=0$ leads to

$A e^{\frac{-i T(1+\sqrt{1-4 E \alpha})}{2 \alpha \hbar}}\left(1-e^{\frac{i T \sqrt{1-4 E \alpha}}{\alpha \hbar}}\right)=0$,

which means that either $A=B=0$ or both the real and the imaginary parts of the above equation are zero. The real part is

$-2 \sin \left(\frac{T}{2 \alpha \hbar}\right) \sin \left(\frac{T \sqrt{1-4 E \alpha}}{2 \alpha \hbar}\right)=0$.

The imaginary part is

$-2 \cos \left(\frac{T}{2 \alpha \hbar}\right) \sin \left(\frac{T \sqrt{1-4 E \alpha}}{2 \alpha \hbar}\right)=0$

If both are zero, then

$\sin \left(\frac{T \sqrt{1-4 E \alpha}}{2 \alpha \hbar}\right)=0$,

leading to

$\frac{T \sqrt{1-4 E \alpha}}{2 \alpha \hbar}=n \pi$,

where $n \in Z$. This means that

$T=n \pi \frac{2 \alpha \hbar}{\sqrt{1-4 E \alpha}}$,

or expanding in terms of $\alpha$ 
$T=2 n \pi \hbar\left(\alpha+2 E \alpha^{2}+6 E^{2} \alpha^{3}+\mathscr{O}\left(\alpha^{4}\right)\right)$

i.e. we can only measure time in discrete steps. It is interesting to note that this discrete interval is dependent on the energy of the system, i.e. the larger the energy the larger will be this discrete interval of time, but since the energy dependence is to second and higher orders, this does not change the time interval by much, except near Planckian energy scales. It may also be noted that this time interval is of the same order as the minimum time expected directly from the time-energy uncertainty in Eq. (9). Further, it appears from Eq. (17) that the minimum time interval diverges as the energy approaches Planck scale $(E \sim 1 / 4 \alpha)$. However, this divergence could be unphysical since the Schrödinger equation (10) is deformed to first order in $\alpha$ only. Finally, as expected, a continuous time is recovered in the limit in which $\alpha \rightarrow 0$. In short, any physical system with finite energy can only evolve by taking discrete jumps in time rather than continuously.

\section{Rate of spontaneous emission}

We now apply the above to a concrete quantum mechanical system. The rate of spontaneous emission in a two-level system is well understood [53]. Here we shall repeat this analysis for a deformed quantum mechanical system. Now for a twolevel system with eigenstates $\psi_{a}$ and $\psi_{b}$, the eigenvalues of the unperturbed Hamiltonian $H^{0}$ can be written as

$H^{0} \psi_{a}=E_{a} \psi_{a}, \quad H^{0} \psi_{b}=E_{b} \psi_{b}$

Any state can be written as a superposition of those eigenstates with the time dependence found in Eq. (11)

$\Psi(t)=c_{a} \psi_{a} e^{\frac{-i t}{2 \alpha \hbar}\left(1-\sqrt{1-4 \alpha E_{a}}\right)}+c_{b} \psi_{b} e^{\frac{-i t}{2 \alpha \hbar}\left(1-\sqrt{1-4 \alpha E_{b}}\right)}$.

If a time-dependent perturbation $H^{\prime}(t)$ was turned on, the wave function $\Psi(t)$ can still be expressed as the previous equation but with a time-dependent $c_{a}(t)$ and $c_{b}(t)$, and the goal is to solve for $c_{a}(t)$ and $c_{b}(t)$. This will also hold if the time evolution of the system is given by a deformed Schrödinger equation. So, let us assume that this system actually evolves according to the deformed time-dependent Schrödinger equation,

$$
\begin{aligned}
H \psi & =H^{0} \psi+H^{\prime}(t) \psi \\
& =i \hbar \partial_{t} \psi+\hbar^{2} \alpha \partial_{t}^{2} \psi .
\end{aligned}
$$

Now neglecting terms of order $\hbar \alpha$ and $\hbar^{2} \alpha$ for a two-level system, we obtain

$$
\begin{aligned}
c_{a} & H^{0} \psi_{a} e^{-i \epsilon_{a} t / \hbar}+c_{b} H^{0} \psi_{b} e^{-i \epsilon_{b} t / \hbar}+c_{a} H^{\prime} \psi_{a} e^{-i \epsilon_{a} t / \hbar} \\
& +c_{b} H^{\prime} \psi_{b} e^{-i \epsilon_{b} t / \hbar} \\
= & i \hbar\left(\dot{c}_{a} \psi_{a} e^{-i \epsilon_{a} t / \hbar}+\dot{c}_{b} \psi_{b} e^{-i \epsilon_{b} t / \hbar}\right)+c_{a} E_{a} \psi_{a} e^{-i \epsilon_{a} t / \hbar} \\
& +c_{b} E_{b} \psi_{b} e^{-i \epsilon_{b} t / \hbar} .
\end{aligned}
$$

To simplify that last expression, we defined

$\epsilon_{a}=\frac{1}{2 \alpha}\left(1-\sqrt{1-4 \alpha E_{a}}\right)$,

$\epsilon_{b}=\frac{1}{2 \alpha}\left(1-\sqrt{1-4 \alpha E_{b}}\right)$.

It may be noted that in the limit $\alpha \rightarrow 0$, we obtain $\epsilon_{a} \rightarrow$ $E_{a}$ and $\epsilon_{b} \rightarrow E_{b}$. The first two terms cancel the last two terms. Now taking the inner product with $\psi_{a}$ and solving for $\dot{c}_{a}$, we obtain

$\dot{c}_{a}=-\frac{i}{\hbar}\left(c_{a} H_{a a}^{\prime}+c_{b} H_{a b}^{\prime} e^{-i \omega_{0} t}\right)$.

Here we have defined

$$
\begin{aligned}
H_{i j}^{\prime} & =\left\langle\psi_{i}\left|H^{\prime}\right| \psi_{j}\right\rangle, \\
\omega_{0} & =\frac{\epsilon_{b}-\epsilon_{a}}{\hbar} \\
& =\frac{\sqrt{1-4 \alpha E_{a}}-\sqrt{1-4 \alpha E_{b}}}{2 \alpha \hbar} .
\end{aligned}
$$

Similarly, the inner product with $\psi_{b}$ picks out $\dot{c}_{b}$,

$\dot{c}_{b}=-\frac{i}{\hbar}\left(c_{b} H_{b b}^{\prime}+c_{a} H_{b a}^{\prime} e^{i \omega_{0} t}\right)$.

Since in most applications the diagonal elements of $H^{\prime}$ vanish, we get the simplified equations

$\dot{c}_{a}=-\frac{i}{\hbar} H_{a b}^{\prime} e^{-i \omega_{0} t} c_{b}, \quad \dot{c}_{b}=-\frac{i}{\hbar} H_{b a}^{\prime} e^{i \omega_{0} t} c_{a}$.

These equations have the same form as the un-deformed two-level system, except that in these equations $\omega_{0}$ is modified. Thus, the standard analysis for the un-deformed twolevel system also holds for a deformed two-level system. So if an atom is exposed to a sinusoidally oscillating electric field $\mathbf{E}=E_{0} \cos (\omega t) \hat{k}$, then the perturbation Hamiltonian can be written as

$H^{\prime}(t)=-q E_{0} \mathbf{r} \cos (\omega t)$

and

$H_{b a}^{\prime}=-\mathbf{p} E_{0} \cos (\omega t)$

where $\mathbf{p}=q\left\langle\psi_{b}|\mathbf{r}| \psi_{a}\right\rangle$ is the electric dipole radiation. Repeating the analysis for the un-deformed two-level system [53], we can write the rate of spontaneous emission $\mathscr{A}$ for the deformed system as 
$\mathscr{A}=\frac{\omega_{0}^{3}|\mathbf{p}|^{2}}{3 \pi \epsilon_{0} \hbar}$.

Expanding to first order in $\alpha$, we obtain

$\mathscr{A}=\frac{\left(E_{b}-E_{a}\right)^{3}|\mathbf{p}|^{2}}{3 \pi \epsilon_{0} \hbar^{4}}+\frac{\left(E_{b}-E_{a}\right)^{3}\left(E_{a}+E_{b}\right)|\mathbf{p}|^{2}}{\pi \epsilon_{0} \hbar^{4}} \alpha$.

To get an order of magnitude estimate of the effect of the extra term in Eq. (31), we consider the spontaneous emission from a transition between the first and second energy levels in the hydrogen atom. Now for these levels, we have $E_{1}=13.6$ $\mathrm{eV}, E_{2}=E_{1} / 4$, and $|\mathbf{p}| \sim 0.7 q a_{0}$, where $a_{0}$ is the Bohr radius. Thus, we obtain

$$
\begin{aligned}
\mathscr{A} & \approx 2.1+1.7 \times 10^{-17} \alpha\left[\mathrm{m}^{-1}\right] \\
& \approx 6.2 \times 10^{8}+5.1 \times 10^{-9} \alpha\left[\mathrm{s}^{-1}\right] .
\end{aligned}
$$

The uncertainty in measuring the rate of spontaneous emission for hydrogen atom is $\pm 0.3 \%$ [54]. So, the bound on $\alpha_{0}$ from the rate of spontaneous emission in a hydrogen atom is given by

$\alpha_{0}<7.2 \times 10^{23}$.

Hence, at this scale the effect of the rate of spontaneous emission in hydrogen can be effected by the temporal deformation proposed in this paper. If such a deformation scale exists at this scale in nature, future measurements might be able to detect it.

It may be noted that we can also use the lifetime of particles to set bounds on $\alpha_{0}$, for the modified Schrödinger equation. For example, the tau has a lifetime of $(290.3 \pm 0.5) \times 10^{-15} \mathrm{~s}$ [55], and since the minimum time from Eq. (18) must be less than the uncertainty in measuring the tau's life time, then $2 \pi \hbar \alpha<0.5 \times 10^{-15} \mathrm{~s}$ This means that $\alpha_{0}<1.5 \times 10^{27}$. However, the bound on $\alpha_{0}$ from the hydrogen atom is more stringent than the bound on $\alpha_{0}$ from the lifetime of particles. So, in the case that a minimum measurable time exists in nature, we are more likely to first observe its effects on the rate of spontaneous emission in hydrogen atoms.

\section{Conclusions}

We have shown here that the existence of a minimum measurable time scale in a quantum theory naturally leads to the discretization of time. This is similar to the existence of a minimum measurable length scale leading to a discretization of space. Thus, a crystal in time gets naturally formed by the existence of a minimum measurable time scale in the universe. Time crystals have been studied recently for systems in which time reparametrization is broken, just as spatial translation is broken in regular crystals. Time crystals have also been studied earlier for analyzing superconducting rings [39-43]. We also analyzed the effect of such a deformation on the rate of spontaneous emission in a hydrogen atom. It would be interesting to analyze a combination of minimum length and minimum time deformations of quantum mechanics to demonstrate a discretization of space and time in four dimensions. We expect to obtain non-local fractional derivative terms in that case, which may possibly be dealt with using a theory of harmonic extension of functions [56,57], or via the Dirac equation approach [34]. It may be noted that it is conceptually useful to view the minimum measurable time as a component of a minimum Euclidean four volume with complex time, and then analytically continue the results to a Lorentz manifold. However, as we analyzed a system with Galilean symmetry, we did not to go through this procedure.

It is expected that the deformation of the Hamiltonian studied here will affect all physical systems. Thus for example, one can study the decay rates of particle and unstable nuclei using this deformed time evolution, which are expected to change as well. In fact, by fixing the value of this deformation parameter just below the experimentally measured limit, it might be possible to devise tests for detecting such deformation of time evolution of quantum mechanics. The deformed Hamiltonian should affect time-dependent perturbation theory as well. For example, the out-of-equilibrium Anderson model has been studied using the time-dependent density functional theory [58]. This has important applications for time-dependent processes in an open system where different scattering processes take place. This behavior will get modified due to this deformation of quantum mechanics. Similarly the quantum mechanical systems for which the strict adiabatic approximation fails, but which do not escape too far from the adiabatic limit, can be analyzed using a timedependent adiabatic deformation of the theory [59]. It would be interesting to analyze the effect of having a minimum measurable time for such a time-dependent adiabatic deformation of the theory.

Acknowledgments The work of SD is supported by the Natural Sciences and Engineering Research Council of Canada.

Open Access This article is distributed under the terms of the Creative Commons Attribution 4.0 International License (http://creativecomm ons.org/licenses/by/4.0/), which permits unrestricted use, distribution, and reproduction in any medium, provided you give appropriate credit to the original author(s) and the source, provide a link to the Creative Commons license, and indicate if changes were made.

Funded by SCOAP ${ }^{3}$.

\section{References}

1. M. Maggiore, Phys. Lett. B 304, 65 (1993)

2. M.I. Park, Phys. Lett. B 659, 698 (2008) 
3. S. Hossenfelder, Living Rev. Relativ. 16, 2 (2013)

4. D. Amati, M. Ciafaloni, G. Veneziano, Phys. Lett. B 216, 41 (1989)

5. A. Kempf, G. Mangano, R.B. Mann, Phys. Rev. D 52, 1108 (1995)

6. L.N. Chang, D. Minic, N. Okamura, T. Takeuchi, Phys. Rev. D 65 , $125027(2002)$

7. L.N. Chang, D. Minic, N. Okamura, T. Takeuchi, Phys. Rev. D 65 , $125028(2002)$

8. S. Benczik, L.N. Chang, D. Minic, N. Okamura, S. Rayyan, T. Takeuchi, Phys. Rev. D 66, 026003 (2002)

9. P. Dzierzak, J. Jezierski, P. Malkiewicz, W. Piechocki, Acta Phys. Polon. B 41, 717 (2010)

10. L.J. Garay, Int. J. Mod. Phys. A 10, 145 (1995)

11. C. Bambi, F.R. Urban, Class. Quantum Grav. 25, 095006 (2008)

12. K. Nozari, Phys. Lett. B. 629, 41 (2005)

13. A. Kempf, G. Mangano, R.B. Mann, Phys. Rev. D 52, 1108 (1995)

14. A. Kempf, J. Phys. A 30, 2093 (1997)

15. S. Das, E.C. Vagenas, Phys. Rev. Lett. 101, 221301 (2008)

16. J. Magueijo, L. Smolin, Phys. Rev. Lett. 88, 190403 (2002)

17. J. Magueijo, L. Smolin, Phys. Rev. D 71, 026010 (2005)

18. J.L. Cortes, J. Gamboa, Phys. Rev. D 71, 065015 (2005)

19. G. 't Hooft, Class. Quantum Grav. 13, 1023 (1996)

20. V.A. Kostelecky, S. Samuel, Phys. Rev. D 39, 683 (1989)

21. G. Amelino-Camelia, J.R. Ellis, N.E. Mavromatos, D.V. Nanopoulos, S. Sarkar, Nature 393, 763 (1998)

22. R. Gambini, J. Pullin, Phys. Rev. D 59, 124021 (1999)

23. S.M. Carroll, J.A. Harvey, V.A. Kostelecky, C.D. Lane, T. Okamoto, Phys. Rev. Lett. 87, 141601 (2001)

24. M. Faizal, Phys. Lett. B 705, 120 (2011)

25. M. Faizal, Mod. Phys. Lett. A 27, 1250075 (2012)

26. M. Faizal, J. Phys. A 44, 402001 (2011)

27. P. Horava, Phys. Rev. D 79, 084008 (2009)

28. J. Magueijo, L. Smolin, Class. Quantum Grav. 21, 1725 (2004)

29. J.J. Peng, S.Q. Wu, Gen. Relativ. Gravit. 40, 2619 (2008)

30. A.F. Ali, M. Faizal, M.M. Khalil, Nucl. Phys. B 894, 341 (2015)

31. A.F. Ali, M. Faizal, M.M. Khalil, Phys. Lett. B 743, 295 (2015)

32. A.F. Ali, M. Faizal, M.M. Khalil, JHEP 1412, 159 (2014)

33. A.F. Ali, Phys. Rev. D 89, 104040 (2014)
34. S. Das, E.C. Vagenas, A.F. Ali, Phys. Lett. B 690, 407 (2010)

35. P. Pedram, K. Nozari, S.H. Taheri, JHEP. 1103, 093 (2011)

36. A.F. Ali, S. Das, E.C. Vagenas, Phys. Rev. D 84, 044013 (2011)

37. A.F. Ali, S. Das, E.C. Vagenas, Phys. Lett. B 678, 497 (2009)

38. M. Faizal, A. F. Ali. S. Das, arXiv:1411.5675 (2014)

39. P. Bruno, Phys. Rev. Lett. 111, 070402 (2013)

40. F. Wilczek, Phys. Rev. Lett. 109, 160401 (2012)

41. A. Shapere, F. Wilczek, Phys. Rev. Lett. 109, 160402 (2012)

42. E. Castillo, B. Koch, G. Palma, arXiv:1410.2261 (2014)

43. H. Watanabe, M. Oshikawa, Phys. Rev. Lett. 114, 251603 (2015)

44. W. Paili, General Principle of Quantum Theory (Springer, Berlin, 1980)

45. J. von Neumann, Mathematische Grundlagen der Quantenmechanik (Springer, Berlin, 1932)

46. P. Buscha, M. Grabowskib, P.J. Lahtic, Phys. Lett. A 191, 357 (1994)

47. V.S. Olkhovsky, Adv. Math. Phys. 2009, 859710 (2009)

48. V.S. Olkhovsky, Int. J. Mod. Phys. A 22, 5063 (2007)

49. V.S. Olkhovsky, E. Recami, Int. J. Mod. Phys. B 22, 1877 (2008)

50. R. Brunetti, K. Fredenhagen, M. Hoge, Found. Phys. 40, 1368 (2010)

51. G. Ludwig, Foundations of Quantum Mechanics, vol. 1 (Springer, Berlin, 1983)

52. A.S. Holevo, Probabilistic and Statistical Aspects of Quantum Theory (North-Holland, Amsterdam, 1982)

53. D.J. Griffiths, Introduction to Quantum Mechanics, 2nd edn. (Prentice Hall, NJ, 2004)

54. W. Wiese, J. Phys. Chem. Ref. Data 38, 565 (2009)

55. K.A. Olive et al. (Particle Data Group), Chin. Phys. C 38, 090001 (2014)

56. M. Faizal, arXiv:1406.2653 (2014)

57. M. Faizal, Int. J. Geom. Methods Mod. Phys. 12, 1550022 (2015)

58. A.M. Uimonen, E. Khosravi, A. Stan, G. Stefanucci, S. Kurth, R. van Leeuwen, E.K.U. Gross, Phys. Rev. B 84, 115103 (2011)

59. D. Viennot, J. Phys. A 47, 065302 (2014) 